\title{
Genetic Factors, Adherence to Healthy Lifestyle Behavior, and Risk of Invasive Breast Cancer Among Women in the UK Biobank
}

\author{
Rhonda S. Arthur (D, , Tao Wang, Xiaonan Xue, Victor Kamensky, Thomas E. Rohan \\ Affiliations of authors: Albert Einstein College of Medicine, Bronx, NY, USA
}

${ }^{*}$ Correspondence to: Rhonda Arthur, PhD, Department of Epidemiology and Population Health, Albert Einstein College of Medicine, 1300 Morris Park Ave, Bronx, NY 10461, (e-mail: rhonda.arthur@einsteinmed.org).

\begin{abstract}
Background: Breast cancer is considered to result from a combination of genetic and lifestyle-related factors, but the degree to which an overall healthy lifestyle may attenuate the impact of multiple genetic variants on invasive breast cancer risk remains equivocal.

Methods: Using Cox proportional hazards regression models, we examined the association of a modified healthy lifestyle index (HLI) with risk of invasive breast cancer by genetic risk group among 146326 women from the UK Biobank. We generated an HLI score based on a combination of diet, physical activity, smoking, alcohol consumption and anthropometry, and a polygenic risk score (PRS) using 304 breast cancer-associated genetic loci.

Results: Among premenopausal and postmenopausal women, a favorable lifestyle (highest tertile) was associated with $22 \%$ and $31 \%$ reductions in invasive breast cancer risk, respectively (hazard ratio $[\mathrm{HR}]_{\text {high vs low }}=0.78,95 \%$ confidence interval $[\mathrm{CI}]$ $=0.64$ to $0.94 ; \mathrm{HR}_{\text {high vs low }}=0.69,95 \% \mathrm{CI}=0.63$ to 0.77 , respectively), whereas a high PRS (highest tertile) was associated with more than a doubling in the risk in both groups. For premenopausal women, the greatest risk reduction in association with the HLI was seen among those with a high PRS $\left(\mathrm{HR}_{\text {high vs low }}=0.73,95 \% \mathrm{CI}=0.75\right.$ to 0.95$)$. In postmenopausal women, those with a favorable lifestyle had $30 \%, 29 \%$, and $32 \%$ reductions in risk of invasive breast cancer in the low, intermediate, and high PRS groups, respectively ( $\mathrm{HR}_{\text {high vs low }}=0.70,95 \% \mathrm{CI}=0.56$ to 0.88 ; $\mathrm{HR}_{\text {high vs low }}=0.71,95 \% \mathrm{CI}=0.59$ to 0.84 ; and $\mathrm{HR}$ high vs low $=0.68,95 \% \mathrm{CI}=0.59$ to 0.78 , respectively). There was an additive but not multiplicative interaction between the HLI score and PRS for postmenopausal and, to a lesser extent, premenopausal women.

Conclusion: Our findings support the view that an overall healthy lifestyle may attenuate the impact of genetic factors on invasive breast cancer risk among women of European ancestry.
\end{abstract}

Breast cancer is a complex disease resulting from a combination of genetic and environmental factors (1). With respect to genetic factors, a few inherited mutations with moderate to high penetrance (eg, BReast CAncer type 1 [BRCA1] and BReast CAncer type 2 [BRCA2]) have been identified (2), but these genetic changes account for only a small proportion of breast cancer cases $(<10 \%)$ (1). Recent advances in breast cancer genetics have led to the identification of many low-penetrance single nucleotide polymorphisms (SNPs), which individually have relatively weak associations with risk of breast cancer (38). However, studies that have examined risk in relation to combinations of breast cancer-associated SNPs have reported much stronger associations (3-8), with pooled findings from 10 studies nested within the Breast Cancer Association Consortium (BCAC) using a 313-SNP polygenic risk score (PRS) and results from the UK Biobank using a 306-SNP PRS showing that the increase in risk of breast cancer per SD increase in the PRS was about $60 \%$ (8).

Several lifestyle-related factors, including obesity (postmenopausal women only), excess alcohol consumption, physical inactivity, and, to some degree, smoking and an unhealthy dietary pattern, have also been positively associated with risk of breast cancer (9). Because these lifestyle factors tend to coexist, a number of recent studies have examined the association of combinations of lifestyle factors with breast cancer risk using various lifestyle indices, and they have provided evidence to support a reduction in risk for those with an overall healthy lifestyle (10-16). Moreover, existing evidence suggests that up to $50 \%$ of breast cancer cases may be preventable by adherence to healthy lifestyle practices $(17,18)$.

Recent studies have indicated that adherence to a healthy lifestyle plays a pivotal role in attenuating the impact of genetic 
factors on risk of several chronic diseases, including coronary heart disease (19), stroke (20), hypertension (21), dementia (22), and colorectal cancer (23). However, to date, no studies to our knowledge have examined whether a combined lifestyle score attenuates the impact of a combination of breast cancerassociated genes on invasive breast cancer risk. Hence, in the study reported here, we examined whether the associations between an overall healthy lifestyle and risk of invasive breast cancer vary by genetic risk categories among women participating in the UK Biobank study.

\section{Methods}

\section{Study Population and Design}

The full UK Biobank study protocol is publicly available (http:// www.ukbiobank.ac.uk/wp-content/uploads/2011/11/UK-BiobankProtocol.pdf). Briefly, the UK Biobank is a large prospective observational study comprising approximately 500000 men and women ( $N=229134$ men, $N=273402$ women) of various ethnicities aged 40-69 years at enrollment. Participants were recruited from across 22 centers located throughout England, Wales, and Scotland between 2006 and 2010 (24,25). The study was established to provide a resource for the investigation of the genetic, environmental, and lifestyle factors associated with a wide range of diseases, including cancer. The study was approved by the North West Multi-center Research Ethics Committee, the National Information Governance Board for Health and Social Care in England and Wales, and the Community Health Index Advisory Group in Scotland (http://www.ukbiobank.ac.uk/ ethics/). All participants provided written informed consent.

\section{Exposure and Covariate Ascertainment}

At enrollment, information on the participants' sociodemographic characteristics, health and medical history, and diet and lifestyle was collected using a self-administered touchscreen questionnaire (http://www.ukbiobank.ac.uk/resources/) and nurse-led interviews. During the interviews, the participants' body weight, waist circumference (WC), and height were measured by trained staff using standardized procedures. Regarding menopausal status, women who reported at recruitment that they had had a menstrual period in the preceding year were classified as premenopausal, and those who reported that they had stopped menstruating at least 1 year before enrollment were classified as postmenopausal. Women who had missing information on menopausal status were classified as postmenopausal if they had had a bilateral oophorectomy (with or without a hysterectomy) or were older than 55 years of age (with or without a hysterectomy and/or bilateral oophorectomy) (26).

\section{Healthy Lifestyle Index (HLI)}

We created a modified HLI score based on the new World Cancer Research Fund/American Institute of Cancer Research (WCRF/AICR) lifestyle score (27). In this study, only five of the eight WCRF/AICR cancer prevention recommendations relating to lifestyle factors were used to generate the HLI score because the UK Biobank food frequency questionnaire did not collect information on the remaining recommendations (see Supplementary Table 1, available online) (27). Specifically, the score included diet, alcohol consumption, physical activity, body mass index (BMI; postmenopausal women only), and WC (postmenopausal women only); the score also included smoking, although this was not included in the WCRF/AICR recommendations (see the Supplementary Methods section, available online, for further details). The HLI score was categorized as low (lowest tertile), intermediate (second tertile), and high (highest tertile) (tertiles were created based on the distribution of the HLI score among the noncases).

\section{Polygenic Risk Score}

A brief description of the procedure for genotyping and imputation of the SNPs is provided in the Supplementary Methods (available online). In a recently published study by Mavaddat et al. (8), a 313 SNP PRS was found to have the best performance compared with the commonly used 77-SNP PRS and with a score based on 3820 SNPs. Thus, for the purpose of this study, we focused on the 313-SNP PRS (see Supplementary Table 2, available online). In the UK Biobank imputed database, only 304 of the 313 SNPs were available for our analyses (8). Detailed information on the generation of the PRS score is provided in the Supplementary Methods (available online).

\section{Outcome Ascertainment}

The outcome for this study was incident invasive breast cancer. In UK Biobank, cancer diagnoses were ascertained through linkage to national cancer registries in England, Wales, and Scotland. Invasive breast cancer was coded using the 10th Revision of the International Classification of Diseases. Death was ascertained via linkage to death registries. Complete follow-up was available through March 31, 2016, for England and Wales and October 31, 2015, for Scotland.

\section{Analytical Cohort}

The study reported here was restricted to women of European ancestry. The process for construction of the analytical cohort can be found in Supplementary Figure 1 (available online). After exclusions, the study population comprised 146326 women.

\section{Statistical Analyses}

Cox proportional hazards models were used to assess the associations of lifestyle factors and the PRS with invasive breast cancer risk with time to diagnosis as the underlying timescale. Analyses were performed separately for premenopausal and postmenopausal women. Women who died, withdrew from the study before the end of follow-up, or had not developed invasive breast cancer by the end of follow-up were censored (ie, noncases). Participants were followed up from their date of enrollment until the date of diagnosis of invasive breast cancer, date of withdrawal from the study, date of death, or until the end of follow-up, whichever came first. Covariates were included in the models if they are known a priori to be associated with risk of invasive breast cancer or if adjustment for them resulted in a $10 \%$ change in the hazard ratio (HR). Regression models were adjusted for age at recruitment, socioeconomic status [based on Townsend deprivation index as quintiles (21)], age at menarche, parity and age at first live birth (as a combined variable), ever use of hormone replacement therapy (HRT; postmenopausal women only), ever use of oral contraceptives, history of mammograms, age at menopause (postmenopausal 
women only), family history of breast cancer, BMI (premenopausal women only), and the first five genetic principal components and genotyping batch, as well as PRS and HLI, unless (for the latter two variables) they were included as the main exposure. Use of Schoenfeld residuals showed that the proportional hazards assumption was not violated. $P_{\text {trend }}$ in risk with the modified HLI score were estimated by including the ordinal HLI variable as a continuous variable in the regression models and testing its coefficient using the Wald test. To test for multiplicative interaction between the lifestyle score and the PRS, we included an interaction term in the regression models and tested its coefficient using the Wald test. Using Cox proportional hazards regression models, we estimated the relative excess risk because of interaction and its $95 \%$ confidence interval (CI) to test for statistical significance of additive interactions, as previously described (28). The additive interaction between the modified HLI and the PRS in association with invasive breast cancer risk was assessed by testing whether the estimated joint effect (ie, relative risk) of the two exposures was greater than the sum of the individual effect estimates for the modified HLI and the PRS.

To address the possibility of reverse causality, we performed sensitivity analyses in which women who were diagnosed with invasive breast cancer within the first 2 years of follow-up were excluded. We also performed sensitivity analyses to assess the association of a modified HLI score that included BMI and WC with risk of invasive breast cancer among premenopausal women (given that body fat is inversely associated with risk of breast cancer among premenopausal women, the scoring of BMI and WC was the reverse of that used for postmenopausal women; here, the models were not adjusted for BMI or WC).

Except for the test for additive interaction (performed using SAS 9.4; SAS Institute Inc., Cary, NC), all statistical analyses were performed using Stata 15 (StataCorp, College Station, TX). All $P$ values were two-sided, and a $P$ value of less than .05 was considered statistically significant.

\section{Results}

Table 1 shows the distribution of the baseline characteristics of the participants by menopausal status. Among premenopausal and postmenopausal women, compared with noncases, cases were less likely to be in the healthiest behavior category (highest tertile) and were more likely to have a high PRS (highest tertile). After a median follow-up of 7.1 years (interquartile range = 6.3-7.7 years), the incidence rate of invasive breast cancer was 3.0 per 1000 person-years among premenopausal women and 3.6 per 1000 person-years among postmenopausal women (Table 2). The PRS was normally distributed among premenopausal and postmenopausal women (Supplementary Figure 2, available online).

We observed risk gradients for the modified HLI score and PRS with risk of invasive breast cancer among premenopausal and postmenopausal women (Figure 1). Specifically, among both groups of women, there was a consistent trend of decreasing risk of breast cancer with increasing HLI score, with premenopausal and postmenopausal women in the high-HLI-score category having $22 \%$ and $31 \%$ reductions in risk of invasive breast cancer, respectively, compared with those in the lowHLI-score category $\left(\mathrm{HR}_{\text {high vs low }}=0.78,95 \% \mathrm{CI}=0.64\right.$ to 0.94 ; $\mathrm{HR}_{\text {high vs low }}=0.69,95 \% \mathrm{CI}=0.63$ to 0.77 , respectively). In contrast, the PRS had a strong positive association with invasive breast cancer risk, with a relatively high PRS being associated with 2.64- and 2.5-fold increases in risk of invasive breast cancer among premenopausal and postmenopausal women, respectively (Figure 1).

The associations between the modified HLI score and risk of invasive breast cancer by PRS categories are shown in Table 3 and Figure 2. Among premenopausal women, the greatest reduction in risk of invasive breast cancer in association with the modified HLI score was observed among those with a high PRS $\left(\mathrm{HR}_{\text {high vs low }}=0.73,95 \% \mathrm{CI}=0.75\right.$ to 0.95 ; Table 3$)$. After the inclusion of BMI and WC in the HLI score, these associations were slightly attenuated among premenopausal women with intermediate and high genetic risk (Supplementary Table 3, available online). In postmenopausal women, those with a favorable lifestyle had $30 \%, 29 \%$, and $32 \%$ reductions in risk of invasive breast cancer in the low, intermediate, and high PRS groups, respectively $\left(\mathrm{HR}_{\text {high vs low }}=0.70,95 \% \mathrm{CI}=0.56\right.$ to $0.88 ; \mathrm{HR}_{\text {high }}$ vs low $=$ $0.71,95 \% \mathrm{CI}=0.59$ to 0.84 ; and $\mathrm{HR}_{\text {high vs low }}=0.68,95 \% \mathrm{CI}=0.59$ to 0.78 , respectively) (Table 3$)$. Compared with those with a low genetic risk and a high HLI score, premenopausal and postmenopausal women with a high genetic risk and a low HLI score had approximately 3.40- and 3.66-fold increases in the risk of invasive breast cancer, respectively (Figure 2). We did not observe a multiplicative interaction between the HLI score and the PRS. However, we observed positive additive interactions for a low modified HLI score with a high PRS for premenopausal women and for intermediate HLI and low HLI scores with high PRS among postmenopausal women. The $P$ values for the overall tests of additive interaction were .06 and .02 for premenopausal and postmenopausal women, respectively (Table 4).

The observed associations between the modified HLI score and invasive breast cancer risk among premenopausal and postmenopausal women did not change after exclusion of women who had a breast cancer diagnosis within the first 2 years of follow-up (Table 5).

Table 6 and Supplementary Figures 3 and 4 (available online) present the associations of the individual HLI components with risk of invasive breast cancer among premenopausal and postmenopausal women. In premenopausal women, only diet and physical activity were associated with altered risk (inverse associations). However, among postmenopausal women, relatively high alcohol consumption, excess adiposity (overall or central), and current smoking were positively associated with risk, whereas physical activity (highest quintile) was inversely associated with risk.

\section{Discussion}

In this large prospective study, we observed that women of European descent with a relatively high PRS had more than a doubling in the risk of invasive breast cancer, whereas an overall healthy lifestyle was associated with a moderate (approximately $27 \%$ ) reduction in the risk of invasive breast cancer. Further, when examining the joint associations of the modified HLI and the PRS with risk, we demonstrated that among premenopausal women, the greatest relative reduction in risk in association with an overall healthy lifestyle occurred among those with high genetic risk. For postmenopausal women, the reduction in risk of breast cancer in association with the modified HLI was similar across all strata of the PRS. Our study also demonstrated a modest additive interaction between these scores among premenopausal and, in particular, postmenopausal women. 
Table 1. Baseline characteristics of women from the UK Biobank by menopausal status

\begin{tabular}{|c|c|c|c|c|}
\hline \multirow[b]{2}{*}{ Characteristics } & \multicolumn{2}{|c|}{ Premenopausal } & \multicolumn{2}{|c|}{ Postmenopausal } \\
\hline & $\begin{array}{c}\text { Cases } \\
(\mathrm{N}=706)\end{array}$ & $\begin{array}{l}\text { Noncases } \\
(\mathrm{N}=34751)\end{array}$ & $\begin{array}{c}\text { Cases } \\
(\mathrm{N}=2716)\end{array}$ & $\begin{array}{c}\text { Noncases } \\
(\mathrm{N}=108153)\end{array}$ \\
\hline Age at enrollment, median (IQR), y & $46(43-48)$ & $45(43-48)$ & $61(58-64)$ & $61(56-64)$ \\
\hline Socioeconomic status, lowest quintile, $\%$ & 21.8 & 20.0 & 20.2 & 20.0 \\
\hline Family history of breast cancer, $\%$ & 15.0 & 10.0 & 15.5 & 11.5 \\
\hline Age at menarche, median (IQR), y & $13(12-14)$ & $13(12-14)$ & $13(12-14)$ & $13(12-14)$ \\
\hline Nulliparous, \% & 32.7 & 27.6 & 16.8 & 17.0 \\
\hline Age at menopause, median (IQR), y & - & - & $51(48-53)$ & $50(48-53)$ \\
\hline OC use ever, \% & 91.8 & 91.6 & 78.7 & 79.9 \\
\hline HRT use ever, \% & - & - & 53.3 & 50.1 \\
\hline Mammogram ever, \% & 38.7 & 33.7 & 95.6 & 97.4 \\
\hline \multicolumn{5}{|l|}{ Healthy lifestyle factors } \\
\hline Diet score, highest tertile, $\%$ & 7.9 & 11.7 & 13.8 & 15.0 \\
\hline Alcohol nonusers, \% & 7.1 & 8.3 & 9.3 & 10.5 \\
\hline Physical activity, $\geq 3000$ MET-min/wk, $\%$ & 22.8 & 27.3 & 27.8 & 30.9 \\
\hline BMI, $18.5-24.9 \mathrm{~kg} / \mathrm{m}^{2}, \%$ & 49.9 & 50.1 & 33.8 & 38.5 \\
\hline $\mathrm{WC},<80 \mathrm{~cm}, \%$ & 53.4 & 51.9 & 32.3 & 37.6 \\
\hline Smoking, never, \% & 63.0 & 62.9 & 54.9 & 57.4 \\
\hline \multicolumn{5}{|l|}{ HLI score, ${ }^{*} \%$} \\
\hline Low & 46.9 & 43.5 & 42.5 & 37.2 \\
\hline Intermediate & 29.8 & 28.4 & 35.2 & 34.9 \\
\hline High & 23.4 & 28.1 & 22.4 & 28.0 \\
\hline \multicolumn{5}{|l|}{ PRS, $† \%$} \\
\hline Low & 19.1 & 33.3 & 19.4 & 33.3 \\
\hline Intermediate & 28.9 & 33.3 & 31.0 & 33.3 \\
\hline High & 52.0 & 33.3 & 49.6 & 33.3 \\
\hline
\end{tabular}

${ }^{*}$ Cutpoints: HLI score: less than or equal to 2.5, greater than 2.5-3, greater than 3 and less than or equal to 3, greater than 3-3.75, greater than 3.75, for premenopausal and postmenopausal women, respectively. BMI = body mass index; IQR = interquartile range; HLI = healthy lifestyle index; HRT = hormone replacement therapy; MET $=$ metabolic equivalent; $\mathrm{OC}=$ oral contraceptive; $\mathrm{PRS}=$ polygenic risk score; $\mathrm{WC}=$ waist circumference.

†PRS score: less than or equal to $-0.5008,-0.5007$ to 0.0072 , greater than 0.0072 and less than or equal to $-0.5191,-0.5190$ to -0.0097 , greater than -0.0096 for premenopausal and postmenopausal women, respectively.

Table 2. Incidence rate of invasive breast cancer by modified HLI and PRS category among premenopausal and postmenopausal women in the UK Biobank

\begin{tabular}{|c|c|c|c|c|}
\hline \multirow[b]{2}{*}{ Scores } & \multicolumn{2}{|c|}{ Premenopausal } & \multicolumn{2}{|c|}{ Postmenopausal } \\
\hline & Cases/noncases & Incidence/1000 person-years & Cases/noncases & Incidence/1000 person-years \\
\hline Overall & & 3.0 & & 3.6 \\
\hline \multicolumn{5}{|l|}{ HLI score* } \\
\hline Low & $331 / 15104$ & 3.1 & $1153 / 40205$ & 4.1 \\
\hline Intermediate & $210 / 9865$ & 3.0 & $956 / 37723$ & 3.6 \\
\hline High & $165 / 9782$ & 2.4 & $607 / 30225$ & 2.9 \\
\hline \multicolumn{5}{|l|}{ PRS* } \\
\hline Low & $135 / 11586$ & 1.7 & $528 / 36051$ & 2.1 \\
\hline Intermediate & $204 / 11582$ & 2.5 & $842 / 36055$ & 3.3 \\
\hline High & $367 / 11583$ & 4.5 & 1346/36 047 & 5.3 \\
\hline
\end{tabular}

${ }^{*} \mathrm{HLI}=$ healthy lifestyle index; PRS = polygenic risk score.

Our findings of linear dose-response associations between the 304 SNP PRS score and risk of invasive breast cancer among premenopausal and postmenopausal women, which were independent of lifestyle-related factors and other conventional breast cancer risk factors, are consistent with those of previous studies (3-8). Interestingly, in the current study, the association between the PRS and risk of invasive breast cancer among premenopausal women was somewhat stronger than that for postmenopausal women. This finding is in agreement with existing evidence that indicates that familial or genetic breast cancer typically occurs at an earlier age than sporadic breast cancer
(29). Similar to this study, a number of previous studies, using various lifestyle indices, have consistently shown an inverse association of an overall healthy lifestyle with risk of invasive breast cancer among premenopausal women $(11,30)$ and, in particular, among postmenopausal women (10-15,31).

Having a genetic predisposition substantially increases a woman's lifetime risk of breast cancer $(32,33)$, and there is evidence to suggest that this risk may be increased or decreased according to an individual's lifestyle (34-38). In keeping with this, one early study showed that among BRCA1/2 carriers, physical activity and a normal body weight contributed to 


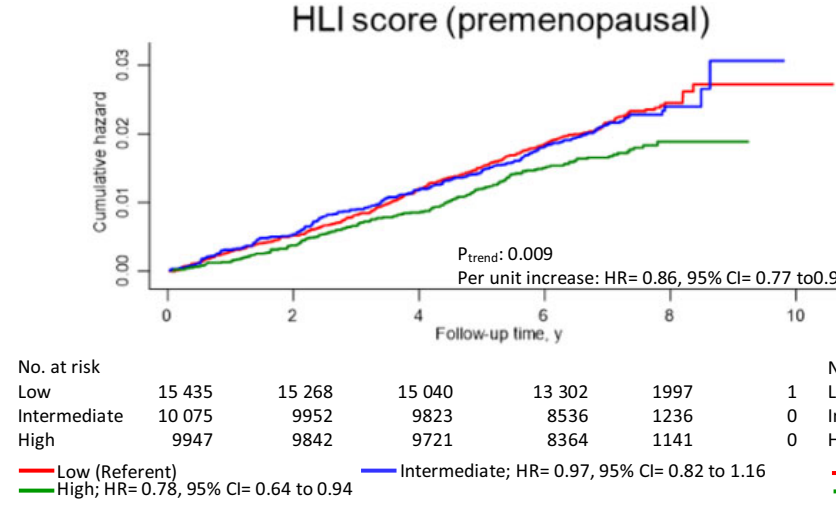

PRS score (premenopausal)
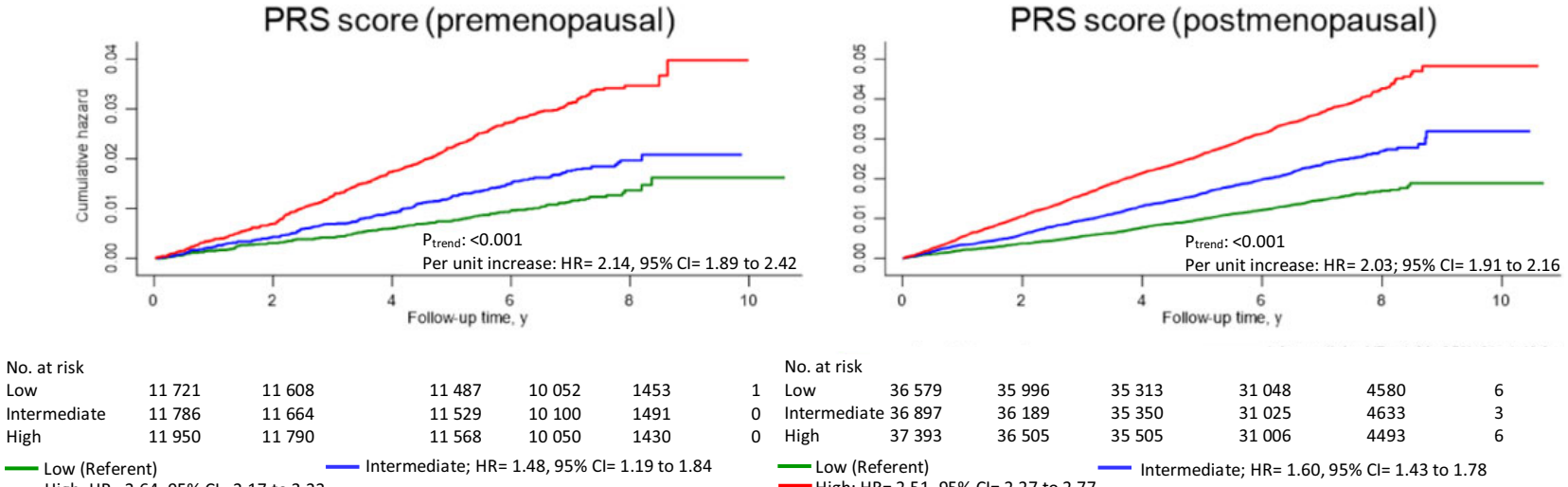

High: $H R=2.64,95 \% \mathrm{Cl}=2.17$ to 3.22

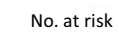

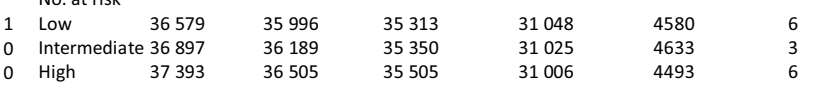

— Low (Referent)

Figure 1. Risk of breastAQ30 cancer by modified healthy lifestyle index (HLI) and polygenic risk score (PRS) among women from the UK Biobank. Adjusted for age at recruitment, socioeconomic status, age at menarche, parity and age at first pregnancy (combined), family history of breast cancer, history of mammograms, oral contraceptive use, age at menopause (postmenopausal women), hormone replacement therapy use (postmenopausal women), body mass index (premenopausal women), first five principal components of ancestry and genotyping batch, lifestyle score, and genetic score, unless included as main exposure, in Cox proportional hazards regression models. Cutpoints: HLI score: less than or equal to 2.5, greater than 2.5 to 3 , greater than 3 and less than or equal to 3 , greater than 3 to 3.75 , greater than 3.75 , for premenopausal and postmenopausal women, respectively; PRS score: less than or equal to $-0.5008,-0.5007$ to 0.0072 , greater than 0.0072 and less than or equal to $-0.5191,-0.5190$ to -0.0097 , greater than -0.0096 for premenopausal and postmenopausal women, respectively. All tests were two-sided. $\mathrm{CI}=$ confidence interval; HR = hazard ratio.

Table 3. Associations between modified HLI and risk of breast cancer by PRS category among women from the UK Biobank*

\begin{tabular}{|c|c|c|c|c|c|c|}
\hline \multirow[b]{2}{*}{ PRS category $†$} & \multicolumn{3}{|c|}{ HLI score $†$} & \multirow[b]{2}{*}{$P_{\text {trend}} \S$} & \multirow[b]{2}{*}{$P_{\text {interaction }} \S$} & \multirow[b]{2}{*}{ Per tertile increase HR $(95 \% \mathrm{CI})^{*}$} \\
\hline & $\begin{array}{c}\text { Low } \\
\text { HR }(95 \% \text { CI })^{*}\end{array}$ & $\begin{array}{l}\text { Intermediate } \\
\mathrm{HR}(95 \% \mathrm{CI})^{*}\end{array}$ & $\begin{array}{c}\text { High } \\
\text { HR }(95 \% \text { CI })^{*}\end{array}$ & & & \\
\hline \multicolumn{7}{|l|}{ Premenopausal $\ddagger$} \\
\hline Low & 1.00 (Referent) & 1.25 (0.84 to 1.86$)$ & 0.97 (0.63 to 1.49$)$ & .96 & .17 & 1.01 (0.80 to 1.28$)$ \\
\hline Intermediate & 1.00 (Referent) & 1.17 (0.85 to 1.60$)$ & $0.76(0.53$ to 1.10$)$ & .18 & & $0.80(0.66$ to 0.98$)$ \\
\hline High & 1.00 (Referent) & 0.80 (0.62 to 1.02$)$ & $0.73(0.57$ to 0.95$)$ & .01 & & 0.85 (0.74 to 0.99$)$ \\
\hline \multicolumn{7}{|l|}{ Postmenopausal } \\
\hline Low & 1.00 (Referent) & $0.90(0.74$ to 1.09$)$ & $0.70(0.56$ to 0.88$)$ & .002 & .85 & 0.88 (0.79 to 0.97$)$ \\
\hline Intermediate & 1.00 (Referent) & 0.82 (0.70 to 0.96$)$ & 0.71 (0.59 to 0.84$)$ & $<.001$ & & 0.83 (0.76 to 0.80$)$ \\
\hline High & 1.00 (Referent) & 0.88 (0.78 to 0.99$)$ & 0.68 (0.59 to 0.78$)$ & $<.001$ & & $0.82(0.77$ to 0.88$)$ \\
\hline
\end{tabular}

*Adjusted for age at recruitment, socioeconomic status, age at menarche, parity and age at first pregnancy (combined), family history of breast cancer, history of mammograms, oral contraceptive use, first five principal components of ancestry and genotyping batch, age at menopause (postmenopausal women only), and hormone replacement therapy use (postmenopausal women only). CI = confidence interval; HLI = healthy lifestyle index; HR = hazard ratio; PRS = polygenic risk score.

†Cutpoints: HLI score: less than or equal to 2.5, greater than 2.5 to 3, greater than 3 and less than or equal to 3 , greater than 3 to 3.75 , greater than 3.75 , for premenopausal and postmenopausal women, respectively. PRS score: less than or equal to $-0.5008,-0.5007$ to 0.0072 , greater than 0.0072 and less than or equal to -0.5191 , -0.5190 to -0.0097 , greater than -0.0096 for premenopausal and postmenopausal women, respectively.

$\ddagger$ Also adjusted for BMI.

$\S$ All tests were two-sided. 


$\begin{array}{lr}\text { High PRS } & \text { Cases/Non- } \\ \text { HLI score } & 186 / 5057 \\ \text { Low } & 94 / 3241 \\ \text { Intermediate } & 87 / 3285 \\ \text { High } & \\ \text { Intermediate PRS } & \\ \text { HLI score } & 90 / 5047 \\ \text { Low } & 71 / 3335 \\ \text { Intermediate } & 43 / 3200 \\ \text { High } & \\ \text { Low PRS } & \\ \text { HLI score } & 55 / 5000 \\ \text { Low } & 45 / 3289 \\ \text { Intermediate } & 35 / 3297 \\ \text { High } & \end{array}$

\section{High PRS \\ HLI score \\ Low}

Intermediate

High

\section{Intermediate PRS}

HLI score

Low

Intermediate

High

Low PRS

HLI score

Low

Intermediate

High

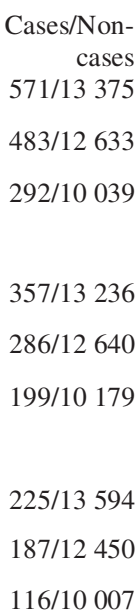

$116 / 10007$

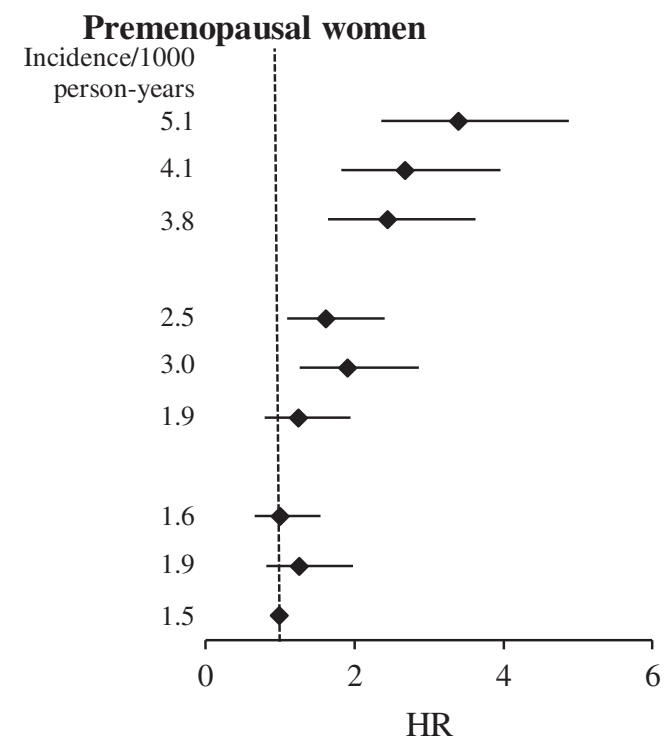

HR $(95 \% \mathrm{CI})$

3.40 (2.36 to 4.88 )

2.69 (1.82 to 3.96$)$

2.45 (1.65 to 3.63$)$

$1.62(1.10$ to 2.41$)$

1.91 (1.27 to 2.86$)$

1.25 (0.80 to 1.95$)$

1.01 (0.66 to 1.55$)$

1.27 (0.82 to 1.98$)$

1.00 (Referent)

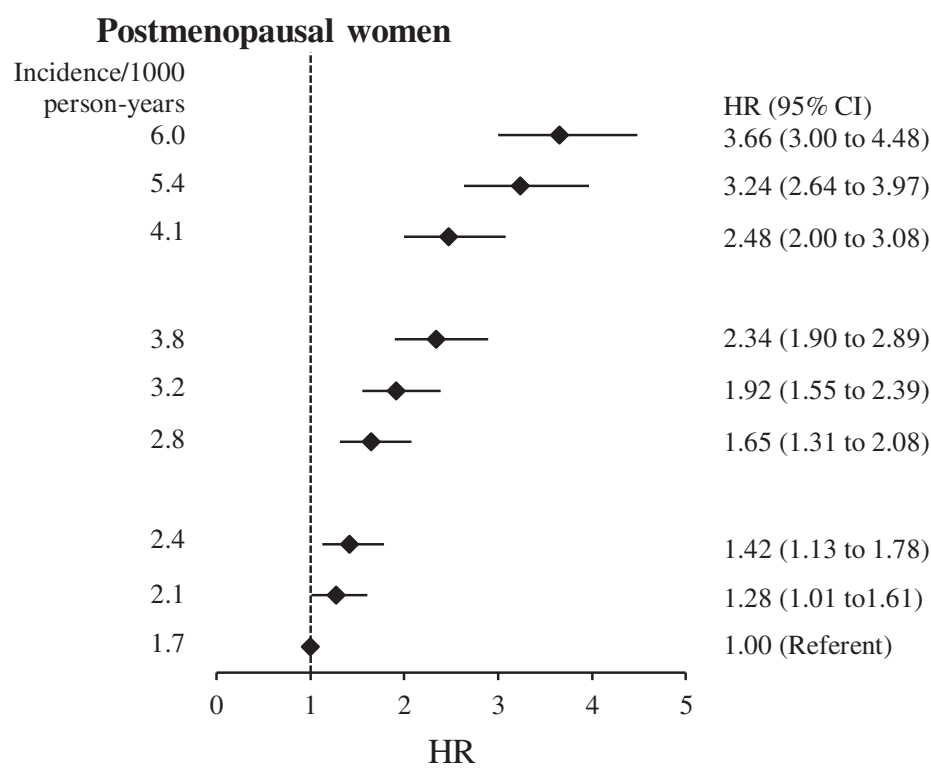

Figure 2. Risk of breast cancer accordingto modified healthy lifestyle index (HLI) and polygenetic risk score (PRS) among women from the UK Biobank. Adjusted for age at recruitment, socioeconomic status, age at menarche, parity and age at first pregnancy (combined), family history of breast cancer, history of mammograms, oral contraceptive use, body mass index (premenopausal women), first five principal components of ancestry and genotyping batch, age at menopause (postmenopausal women only), and hormone replacement therapy use in Cox proportional hazards regression models. Cut points: HLI score: less than or equal to 2.5 , greater than 2.5 to 3, greater than 3 and less than or equal to 3, greater than 3 to 3.75, greater than 3.75, for premenopausal and postmenopausal women, respectively; PRS score: less than or equal to $-0.5008,-0.5007$ to 0.0072 , greater than 0.0072 and less than or equal to $-0.5191,-0.5190$ to -0.0097 , greater than -0.0096 for premenopausal and postmenopausal women, respectively. All tests were two-sided. $\mathrm{CI}=$ confidence interval; $\mathrm{HR}=$ hazard ratio.

delayed age at onset of breast cancer (34). Moreover, other studies have reported an increased risk of breast cancer with smoking, physical inactivity, and high energy intake among BRCA carriers (35-38). Similarly, we observed that an overall healthy lifestyle was associated with a reduction in the risk of breast cancer among premenopausal and postmenopausal women with a strong genetic predisposition (ie, having a high PRS). Further, our study showed that compared with women with a low genetic risk and high modified HLI score, the greatest increase in risk was observed among those with a high genetic risk and low HLI score.
Some studies have observed multiplicative interactions between lifestyle factors, including current smoking (39), alcohol consumption $(40,41)$, BMI $(42)$, and selected breast cancerassociated SNPs. Moreover, in the Breast Cancer Association Consortium study, there was a multiplicative interaction between alcohol consumption and a 77-SNPs PRS (43). In the current study, we did not observe interaction on the multiplicative scale between the modified HLI and the PRS. However, we did observe an additive interaction between the scores for postmenopausal and, to some degree, premenopausal women. The observed additive interactions between the scores suggest that 
Table 4. RERI and 95\% CI for additive interaction between modified HLI and PRS*

\begin{tabular}{|c|c|c|c|c|c|c|}
\hline \multirow[b]{3}{*}{ HLI score $†$} & \multicolumn{6}{|c|}{ PRS† } \\
\hline & \multicolumn{3}{|c|}{ Intermediate } & \multicolumn{3}{|c|}{ High } \\
\hline & RERI* & $95 \% \mathrm{CI}$ & $P \neq$ & RERI* & $95 \% \mathrm{CI}$ & $P \neq$ \\
\hline \multicolumn{7}{|l|}{ Premenopausal§ } \\
\hline Intermediate & 0.41 & $-0.31,1.13$ & .132 & -0.06 & $-0.96,0.84$ & .55 \\
\hline Low & 0.36 & $-0.25,0.98$ & .122 & 0.87 & $0.12,1.63$ & .01 \\
\hline Overall test of all RERI & - & - & - & - & - & .06 \\
\hline \multicolumn{7}{|l|}{ Postmenopausal } \\
\hline Intermediate & -0.03 & $-0.44,0.39$ & .553 & 0.47 & $0.02,0.93$ & .02 \\
\hline Low & 0.23 & $-0.18,0.64$ & .139 & 0.73 & $0.27,1.19$ & .001 \\
\hline Overall test of all RERI & - & - & - & - & - & .02 \\
\hline
\end{tabular}

*Adjusted for age at recruitment, socioeconomic status, age at menarche, parity and age at first pregnancy (combined), family history of breast cancer, history of mammograms, oral contraceptive use, first five principal components of ancestry and genotyping batch, age at menopause (postmenopausal women only), and hormone replacement therapy use (postmenopausal women only). CI = confidence interval; HLI = healthy lifestyle index; PRS = polygenic risk score; RERI = relative excess risk because of interaction (to estimate the RERI, the healthiest lifestyle behavior [high HLI score] and the lowest genetic risk [low PRS] groups were the reference categories)

†Cut points: HLI score: less than or equal to 2.5, greater than 2.5 to 3, greater than 3 and less than or equal to 3 , greater than 3 to 3.75 , greater than 3.75 , for premenopausal and postmenopausal women, respectively; PRS score: less than or equal to $-0.5008,-0.5007$ to 0.0072 , greater than 0.0072 and less than or equal to -0.5191 , -0.5190 to -0.0097 , greater than -0.0096 for premenopausal and postmenopausal women, respectively.

$\ddagger$ All tests were two-sided.

$\S$ Also adjusted for body mass index.

Table 5. Associations between modified HLI and risk of breast cancer by PRS category among women from the UK Biobank (excluding those with a breast cancer diagnosis within 2 years of recruitment)

\begin{tabular}{|c|c|c|c|c|c|}
\hline \multirow[b]{2}{*}{ PRS category§,\| } & \multicolumn{3}{|c|}{ HLI score§ } & \multirow[b]{2}{*}{$P_{\text {trend }} \dagger$} & \multirow[b]{2}{*}{$P_{\text {interaction }} \dagger$} \\
\hline & $\begin{array}{c}\text { Low } \\
\text { HR }(95 \% \mathrm{CI})^{*}\end{array}$ & $\begin{array}{l}\text { Intermediate } \\
\text { HR }(95 \% \text { CI) }\end{array}$ & $\begin{array}{c}\text { High } \\
\text { HR }(95 \% \text { CI)* }\end{array}$ & & \\
\hline \multicolumn{6}{|l|}{ Premenopausal } \\
\hline Low & 1.00 (Referent) & 1.18 (0.74 to 1.88$)$ & 0.95 (0.58 to 1.57$)$ & .93 & .23 \\
\hline Intermediate & 1.00 (Referent) & 1.36 (0.95 to 1.94$)$ & 0.82 (0.53 to 1.26$)$ & .85 & \\
\hline High & 1.00 (Referent) & 0.72 (0.54 to 0.96$)$ & 0.74 (0.56 to 0.97$)$ & .03 & \\
\hline \multicolumn{6}{|l|}{ Postmenopausal } \\
\hline Low & 1.00 (Referent) & $0.90(0.72$ to 1.13$)$ & $0.66(0.50$ to 0.86$)$ & .003 & .85 \\
\hline Intermediate & 1.00 (Referent) & 0.81 (0.67 to 0.97 ) & 0.71 (0.58 to 0.87$)$ & .001 & \\
\hline High & 1.00 (Referent) & 0.84 (0.73 to 0.98$)$ & 0.70 (0.59 to 0.83$)$ & $<.001$ & \\
\hline
\end{tabular}

*Adjusted for age at recruitment, socioeconomic status, age at menarche, parity and age at first pregnancy (combined), family history of breast cancer, history of mammograms, oral contraceptive use, first five principal components of ancestry and genotyping batch, age at menopause (postmenopausal women only), and HRT use. $\mathrm{CI}=$ confidence interval; HLI = healthy lifestyle index; HRT = hormone replacement therapy; PRS = polygenic risk score.

†All tests were two-sided.

$\ddagger$ Also adjusted for body mass index.

§Cut points: HLI score: less than or equal to 2.5 , greater than 2.5 to 3 , greater than 3 and less than or equal to 3 , greater than 3 to 3.75 , greater than 3.75 , for premenopausal and postmenopausal women, respectively. PRS score: less than or equal to $-0.5008,-0.5007$ to 0.0072 , greater than 0.0072 and less than or equal to -0.5191 , -0.5190 to -0.0097 , greater than -0.0096 for premenopausal and postmenopausal women, respectively.

genetic and lifestyle factors may have a stronger joint effect on risk of invasive breast cancer rather than the sum of individual effects of the HLI and PRS. We are not aware of previous studies that have assessed additive interactions between lifestyle and genetic scores in relation to risk of invasive breast cancer. Interaction on the additive scale is rarely assessed in gene-environment studies, but this form of interaction provides an indication of the presence of biological interaction between risk factors (45) and therefore has important etiological implications $(44,46)$. Two risk factors are said to have a biological interaction if both operate in the same pathway towards disease (45). Additive interaction also has important public health implications (46) because it can help to identify groups of individuals who are more likely to benefit from interventions. For example, in this study, among premenopausal women, it would appear that lifestyle intervention would have the greatest impact on those with a high genetic risk. Further studies need to be conducted to confirm our findings.

Strengths of this study include its large sample size, information on a wide range of breast cancer-associated SNPs, the use of standardized protocols for data collection, and ascertainment of cases by linkage to national cancer registries. However, the study also has several limitations. First, some of the lifestyle-related factors were self-reported, and therefore, they may have been misclassified. However, it is likely that misclassification was nondifferential because the participants' disease status was unknown at baseline, so reporting of lifestyle factors is unlikely to have been influenced by the participants' disease status. Such misclassification may have biased the results towards the null. Second, the lifestyle-related factors were based 
Table 6. Associations between modified HLI components and risk of breast cancer among women from the UK Biobank*

\begin{tabular}{|c|c|c|}
\hline HLI components & $\begin{array}{l}\text { Premenopausal } \\
\text { HR }(95 \% \text { CI)* }\end{array}$ & $\begin{array}{c}\text { Postmenopausal } \\
\text { HR }(95 \% \text { CI) }\end{array}$ \\
\hline \multicolumn{3}{|l|}{ Diet score } \\
\hline 0 & 1.00 (Referent) & 1.00 (Referent) \\
\hline 1 & 1.06 (0.90 to 1.26$)$ & 0.97 (0.89 to 1.05$)$ \\
\hline 2 & 0.67 (0.51 to 0.89$)$ & 0.92 (0.82 to 1.04$)$ \\
\hline \multicolumn{2}{|l|}{ Alcohol, g/d } & .18 \\
\hline None & 1.00 (Referent) & 1.00 (Referent) \\
\hline$>0-14$ & 1.06 (0.77 to 1.39$)$ & 1.08 (0.94 to 1.23 ) \\
\hline$>14$ & 1.11 (0.82 to 1.51$)$ & 1.15 (0.99 to 1.33 ) \\
\hline$P_{\text {trend }} \dagger$ & .27 & .07 \\
\hline Per $10 \mathrm{~g} / \mathrm{d}$ & 1.02 (0.95 to 1.08$)$ & 1.05 (1.01 to 1.08 ) \\
\hline \multicolumn{3}{|c|}{ Physical activity MET-min/wk } \\
\hline$<600$ & 1.00 (Referent) & 1.00 (Referent) \\
\hline$\geq 600-<3000$ & 1.03 (0.84 to 1.25$)$ & 0.97 (0.87 to 1.07 ) \\
\hline$\geq 3000$ & 0.85 (0.67 to 1.08$)$ & 0.86 (0.77 to 0.97$)$ \\
\hline$P_{\text {trend }} \dagger$ & .06 & .004 \\
\hline Per 10 MET-min/wk & 0.99 (0.98 to 1.00$)$ & 0.98 (0.97 to 0.99 ) \\
\hline \multicolumn{3}{|l|}{$\mathrm{BMI} \mathrm{kg} / \mathrm{m}^{2}$} \\
\hline $18.5-24.9$ & 1.00 (Referent) & 1.00 (Referent) \\
\hline $25.0-29.9$ & 1.02 (0.86 to 1.20$)$ & 1.17 (1.07 to 1.28 ) \\
\hline$\geq 30$ & 0.89 (0.71 to 1.10$)$ & 1.24 (1.12 to 1.38$)$ \\
\hline$P_{\text {trend }} \dagger$ & 0.33 & $<0.001$ \\
\hline Per $5 \mathrm{~kg} / \mathrm{m}^{2}$ & 0.97 (0.89 to 1.05$)$ & 1.09 (1.05 to 1.13$)$ \\
\hline \multicolumn{3}{|l|}{$\mathrm{WC}, \mathrm{cm}$} \\
\hline$<80$ & 1.00 (Referent) & 1.00 (Referent) \\
\hline$>80-<88$ & 0.89 (0.74 to 1.08$)$ & 1.15 (1.04 to 1.27$)$ \\
\hline$\geq 88$ & 0.94 (0.78 to 1.13 ) & 1.26 (1.15 to 1.38$)$ \\
\hline$P_{\text {trend }} \dagger$ & .39 & $<.001$ \\
\hline Per $10 \mathrm{~cm}$ & 0.97 (0.91 to 1.04$)$ & 1.09 (1.05 to 1.12$)$ \\
\hline \multicolumn{3}{|l|}{ Smoking } \\
\hline Never & 1.00 (Referent) & 1.00 (Referent) \\
\hline Former & 1.01 (0.85 to 1.21$)$ & 1.07 (0.99 to 1.16$)$ \\
\hline Current & 1.10 (0.84 to 1.43$)$ & $1.16(1.002$ to 1.35$)$ \\
\hline$P_{\text {trend }} \dagger$ & .55 & .021 \\
\hline
\end{tabular}

*Adjusted for age at recruitment, socioeconomic status, age at menarche, parity and age at first pregnancy (combined), family history of breast cancer, history of mammograms, oral contraceptive use, age at menopause (postmenopausal women), hormone replacement therapy use (postmenopausal women), first five principal components of ancestry and genotyping batch, genetic score, diet score, alcohol consumption, physical activity, BMI and smoking unless included as main exposure. $\mathrm{BMI}=$ body mass index; $\mathrm{CI}=$ confidence interval; $\mathrm{HLI}=$ healthy lifestyle index; $\mathrm{HR}=$ hazard ratio; $\mathrm{MET}=$ metabolic equivalent; $\mathrm{WC}=$ waist circumference

†All tests were two-sided.

on a single measure; consequently, we were unable to account for the influence of longitudinal changes in these factors on risk of breast cancer. Third, this study was restricted to women of European descent, and therefore the findings may not be generalizable to non-European populations. Further, in the UK Biobank, participants have a healthier lifestyle, lower rates of comorbidities, and, among elderly women, lower all-cause mortality rates than the general population (47). Hence, the findings may not be generalizable to the general UK population due to the presence of healthy volunteer selection bias (47). Last, information on breast cancer stage and hormone-receptor subtype is currently unavailable in the UK Biobank, as a result of which we could not determine whether the observed associations vary by these breast cancer characteristics.

In conclusion, our findings support the view that an overall healthy lifestyle can attenuate the deleterious effect of genetic factors on the risk of invasive breast cancer among women of European descent. Importantly, our findings raise the possibility that among premenopausal women, lifestyle intervention may have the greatest impact on those with a high genetic risk but that among postmenopausal women, lifestyle intervention may be beneficial irrespective of an individual's genetic predisposition.

\section{Funding}

This work was supported by the Breast Cancer Research Foundation (BCRF-16-137 to TER).

\section{Notes}

The funder had no role in the design of the study; the collection, analysis, and interpretation of the data; the writing of the manuscript; and the decision to submit the manuscript for publication. The authors have no disclosures.

This research was conducted using the UK Biobank Resource (Project ID: 40427). We thank the British Association for Cancer Research (BACR) for awarding Rhonda Arthur a BACR travel grant to support her attendance at the AACR Annual Meeting 2019 on March 31, as part of the Minisymposium on Nutrition, Physical Activity, and Cancer Prevention: Current Status and Emerging Strategies where she orally presented results from this study.

\section{References}

1. Song M, Lee K, Kang D. Breast cancer prevention based on gene-environment interaction. Mol Carcinog. 2011;50(4):280-290.

2. De Grève J, Sermijn E, De Brakeleer S, Ren Z, Teugels E. Hereditary breast cancer: from bench to bedside. Curr Opin Oncol. 2008;20(6):605-613.

3. Mavaddat N, Pharoah PDP, Michailidou K, et al. Prediction of breast cancer risk based on profiling with common genetic variants. J Natl Cancer Inst. 2015; 107(5):djv036

4. Shieh Y, Hu D, Ma L, et al. Breast cancer risk prediction using a clinical risk model and polygenic risk score. Breast Cancer Res Treat. 2016;159(3):513-525.

5. Wen W, Shu X, Guo X, et al. Prediction of breast cancer risk based on common genetic variants in women of East Asian ancestry. Breast Cancer Res. 2016; 18(1):124

6. Vachon CM, Pankratz VS, Scott CG, et al. The contributions of breast density and common genetic variation to breast cancer risk. J Natl Cancer Inst. 2015; 107(5):dju397.

7. Cuzick J, Brentnall AR, Segal C, et al. Impact of a panel of 88 single nucleotide polymorphisms on the risk of breast cancer in high-risk women: results from two randomized tamoxifen prevention trials. J Clin Oncol. 2017;35(7):743-750.

8. Mavaddat N, Michailidou K, Dennis J, et al. Polygenic risk scores for prediction of breast cancer and breast cancer subtypes. Am J Hum Genet. 2019;104(1) 21-34.

9. World Cancer Research Fund-American Institute for Cancer Research Continuous update project report. Food, nutrition, physical activity, and the prevention of breast cancer. Wcrf.org/breast-cancer-2017. Published 2017. Accessed May 31, 2017.

10. Thomson CA, McCullough ML, Wertheim BC, et al. Nutrition and physical activity cancer prevention guidelines, cancer risk, and mortality in the Women's Health Initiative. Cancer Prev Res (Phila). 2014;7(1):42-53.

11. Catsburg C, Miller AB, Rohan TE. Adherence to cancer prevention guidelines and risk of breast cancer. Int J Cancer. 2014;135(10):2444-2452.

12. Romaguera D, Gracia-Lavedan E, Molinuevo A, et al. Adherence to nutritionbased cancer prevention guidelines and breast, prostate and colorectal cancer risk in the MCC-Spain case-control study. Int J Cancer. 2017;141(1):83-93.

13. Arthur R, Kirsh VA, Kreiger N, Rohan T. A healthy lifestyle index and its association with risk of breast, endometrial, and ovarian cancer among Canadian women. Cancer Causes Control. 2018;29(6):485-493.

14. Arthur R, Wassertheil-Smoller S, Manson JE, et al. The combined association of modifiable risk factors with breast cancer risk in the Women's Health Initiative. Cancer Prev Res. 2018;11(6):317-326.

15. McKenzie F, Ferrari P, Freisling H, et al. Healthy lifestyle and risk of breast cancer among postmenopausal women in the European Prospective Investigation into cancer and nutrition cohort study. Int J Cancer. 2015; 136(11):2640-2648. 
16. McKenzie F, Ellison-Loschmann L, Jeffreys M, Firestone R, Pearce N, Romieu I Healthy lifestyle and risk of breast cancer for indigenous and nonindigenous women in New Zealand: a case control study. BMC Cancer. 2014; 14(1):12.

17. Colditz GA, Atwood KA, Emmons K, et al. Harvard report on cancer prevention volume 4: Harvard Cancer Risk Index. Cancer Causes Control. 2000;11(6): 477-488.

18. Harvie M, Howell A, Evans DG. Can diet and lifestyle prevent breast cancer: what is the evidence? Am Soc Clin Oncol Educ Book. 2015;35:e66-e73.

19. Khera AV, Emdin CA, Drake I, et al. Genetic risk, adherence to a healthy lifestyle, and coronary disease. N Engl J Med. 2016;375(24):2349-2358.

20. Rutten-Jacobs L, Larsson SC, Malik R, et al. Genetic risk, incident stroke, and the benefits of adhering to a healthy lifestyle: cohort study of 306, 473 UK Biobank participants. BMJ. 2018;363:k4168.

21. Pazoki R, Dehghan A, Evangelou E, et al. Genetic predisposition to high blood pressure and lifestyle factors: associations with midlife blood pressure levels and cardiovascular events. Circulation. 2018;137(7):653-661.

22. Lourida I, Hannon E, Littlejohns T, et al. Association of lifestyle and genetic risk with incidence of dementia. JAMA. 2019;322(5):430.

23. Carr PR, Weigl K, Jansen L, et al. Healthy lifestyle factors associated with lower risk of colorectal cancer irrespective of genetic risk. Gastroenterology. 2018;155(6):1805-1815.e5.

24. Allen N, Sudlow C, Downey P, et al. UK Biobank: current status and what it means for epidemiology. Health Policy Technol. 2012;1(3):123-126.

25. Sudlow C, Gallacher J, Allen N, et al. UK Biobank: an open access resource for identifying the causes of a wide range of complex diseases of middle and old age. PLoS Med. 2015;12(3):e1001779.

26. NHS. Menopause. https://www.nhs.uk/conditions/menopause/. Published 2018. Accessed September 11, 2019.

27. Shams-White M, Brockton TN, Mitrou P, et al. Operationalizing the 2018 World Cancer Research Fund/American Institute for Cancer Research (WCRF/AICR) cancer prevention recommendations: a standardized scoring system. Nutrients. 2019;11(7):pii: E1572.

28. Li R, Chambless L. Test for additive interaction in proportional hazards models. Ann Epidemiol. 2007;17(3):227-236.

29. Easton DF. Familial risks of breast cancer. Breast Cancer Res. 2002;4(5):179-181.

30. Sánchez-Zamorano L, Flores-Luna L, Angeles-Llerenas A, et al. Healthy lifestyle on the risk of breast cancer. Cancer Epidemiol Biomarkers Prev. 2011;20(5): 912-922.

31. Hastert TA, Beresford SAA, Patterson RE, Kristal AR, White E. Adherence to WCRF/AICR cancer prevention recommendations and risk of postmenopausal breast cancer. Cancer Epidemiol Biomarkers Prev. 2013;22(9):1498-1508.

32. Antoniou A, Pharoah PDP, Narod S, et al. Average risks of breast and ovarian cancer associated with BRCA1 or BRCA2 mutations detected in case series unselected for family history: a combined analysis of 22 studies. Am J Hum Genet. 2003;72(5):1117-1130.

33. Chen S, Parmigiani G. Meta-analysis of BRCA1 and BRCA2 penetrance. J Clin Oncol. 2007;25(11):1329-1333.

34. King M, Marks JH, Mandell JB; New York Breast Cancer Study Group. Breast and ovarian cancer risks due to inherited mutations in BRCA1 and BRCA2. Science. 2003;302(5645):643-646.

35. Manders $\mathrm{P}$, Pijpe A, Hooning MJ, et al. Body weight and risk of breast cancer in BRCA1/2 mutation carriers. Breast Cancer Res Treat. 2011;126(1):193-202.

36. Nkondjock A, Robidoux A, Paredes Y, Narod SA, Ghadirian P. Diet, lifestyle and BRCA-related breast cancer risk among French-Canadians. Breast Cancer Res Treat. 2006;98(3):285-294.

37. Ko KP, Kim SJ, Huzarski T, et al. The association between smoking and cance incidence in BRCA1 and BRCA2 mutation carriers. Int J Cancer. 2018;142(11) 2263-2272.

38. Bissonauth V, Shatenstein B, Fafard E, et al. Weight history, smoking, physical activity and breast cancer risk among French-Canadian women noncarriers of more frequent BRCA1/2 mutations. J Cancer Epidemiol. 2009;2009: 748367. doi: 10.1155/2009/748367.

39. Barrdahl M, Rudolph A, Hopper JL, et al. Gene-environment interactions involving functional variants: results from the Breast Cancer Association Consortium. Int J Cancer. 2017;141(9):1830-1840.

40. Travis RC, Reeves GK, Green J, et al. Gene-environment interactions in 7610 women with breast cancer: prospective evidence from the Million Women Study. Lancet. 2010;375(9732):2143-2151.

41. Nickels S, Truong T, Hein R, et al. Evidence of gene-environment interactions between common breast cancer susceptibility loci and established environmental risk factors. PLoS Genet. 2013;9(3):e1003284.

42. Schoeps A, Rudolph A, Seibold P, et al. Identification of new genetic susceptibility loci for breast cancer through consideration of gene-environment interactions. Genet Epidemiol. 2014;38(1):84-93.

43. Rudolph A, Song M, Brook MN, et al. Joint associations of a polygenic risk score and environmental risk factors for breast cancer in the Breast Cancer Association Consortium. Int J Epidemiol. 2018;47(2):526-536.

44. Knol MJ, VanderWeele TJ, Groenwold RHH, Klungel OH, Rovers MM, Grobbee DE. Estimating measures of interaction on an additive scale for preventive exposures. Eur J Epidemiol. 2011;26(6):433-438.

45. Ahlbom A, Alfredsson L. Interaction: a word with two meanings creates confusion. Eur J Epidemiol. 2005;20(7):563-564.

46. VanderWeele TJ, Knol MJ. A tutorial on interaction. Epidemiol Methods. 2014; 3(1):33-72.

47. Fry A, Littlejohns TJ, Sudlow C, et al. Comparison of sociodemographic and health-related characteristics of UK biobank participants with those of the general population. Am J Epidemiol. 2017;186(9):1026-1034. 